\title{
Identificación de flora apícola en el fundo Vitaliano, Amazonas, Perú.
}

\section{Identification of apicultural flora in the Vitaliano farm, Amazonas, Peru.}

\author{
Flor Teresa García Huamán ${ }^{1}$ Marleny Angeles Trauco ${ }^{2}$
}

\section{RESUMEN}

El presente estudio estuvo orientado a identificar la flora apícola del Fundo Vitaliano, ubicado en el distrito de Chachapoyas, departamento de Amazonas, durante diez meses, de febrero a noviembre. El diseño que se utilizó fue el Diseño No Experimental Transaccional, diseño de una sola casilla, se realizaron muestreos de tipo intencional y selectivo, se ubicaron los apiarios existentes y después de localizadas las colmenas se marcaron transeptos radiales y se realizó el inventario de las especies de flora apícola a cada lado del transepto, la época de floración se determinó teniendo en cuenta revisión de fuentes bibliográficas, observaciones directas y encuestas; la identificación taxonómica de los especímenes vegetales se realizó utilizando claves botánicas, información bibliográfica y la respectiva comparación con el material herbarizado en el Herbarium Truxillense de la Universidad Nacional de Trujillo. Se encontró 18 apiarios ubicados a una altitud promedio de 2,434 msnm., 58 especies de flora apícola y las flores tienen en promedio 7.3 grados brix. Se concluyo que existen 24 familias y 48 géneros de flora apícola en el Fundo Vitaliano, la familia que tiene mayor predominancia es la Asteraceae con un $22.4 \%$, las plantas que tienen mayor grado Brix $\left({ }^{\circ} \mathrm{B}\right)$ son Pyrus malus "manzana israel" y Ceasalpina spinosa "tara", con valores de $16^{\circ} \mathrm{B}$ y $15^{\circ} \mathrm{B}$, respectivamente, la flora apícola con diez meses de floración corresponde a Salvia leucantha "salvia morada", Salvia tubiflora "salvia-chochocon" y fragraria vesca "fresa".

Palabras clave: Flora apícola, plantas de uso apícola.

\begin{abstract}
The present study was oriented to identify the beekeeping flora of the Vitaliano Farm, located in the district of Chachapoyas, department of Amazonas, during ten months, from February to November. The design that was used was the Transactional Non-Experimental Design, a single-box design, intentional and selective sampling, the existing apiaries were located and after the hives, radial transepts were marked and the inventory of the species was made of apicultural flora on each side of the transept, the time of flowering was determined taking into account review of bibliographical sources, direct observations and surveys; the taxonomic identification of the plant specimens was done using botanical keys, bibliographic information and the respective comparison with the herbarium material in the Herbarium Truxillense of the National University of Trujillo. It was found 18 apiaries located at an average altitude of 2,434 meters above sea level, 58 species of apicultural flora and flowers have an average of 7.3 degrees Brix. It was concluded that there are 24 families and 48 genera of beekeeping flora in the Vitaliano Farm, the family that has the most predominance is the Asteraceae with $22.4 \%$, the plants that have a higher Brix $\left({ }^{\circ} \mathrm{B}\right)$ are Pyrus malus "Israel apple" and Ceasalpina spinosa "tara", with values of $16^{\circ} \mathrm{B}$ and $15^{\circ} \mathrm{B}$, respectively, the apicultural flora with ten months of flowering corresponds to Salvia leucantha "salvia morada", Salvia tubiflora "salvia-chochocon" and Fragraria vesca "strawberry".
\end{abstract}

Keywords: Apicultural flora, beekeeping plants.

'Docente Principal de la Facultad de Ingeniería y Ciencias Agrarias de la Universidad Nacional Toribio Rodríguez de Mendoza de Amazonas Biólogo-Microbiólogo, Magister en Ciencias con Mención en Gestión Ambiental, Doctora en Ciencias Ambientales y Especialista en Gestión de la Biodiversidad. Universidad Nacional de Trujillo. Correo Electrónico: flor.garcia@untrm.edu.pe

${ }^{2}$ Técnico de Laboratorio Facultad de Ingeniería y Ciencias Agrarias de la Universidad Nacional Toribio Rodríguez de Mendoza de Amazonas. Correo Electrónico: marleny.angeles@untrm.edu.pe 


\section{INTRODUCCIÓN}

El conocimiento de la flora de importancia apícola es fundamental para la conducción racional del apiario ya que constituye el recurso con que cuentan las abejas para alimentarse y producir. La flora es la que define la alternativa productiva (miel, cera, polen, jalea real, propóleos, núcleos, paquetes y reinas), y pone límites a la producción, dependiendo de ella las características del producto. Permite establecer pautas de manejo de las colmenas (Ej.: alimentación suplementaria, incentivación, nucleado, etc.) que optimicen el aprovechamiento de los recursos. Así mismo brinda información para determinar pautas de manejo del apiario en general (ej.: conveniencia y momento de la trashumancia) y aún del campo en que se encuentra ubicado el colmenar. (SAGP, 2012).

Las especies de interés apícola proveen de recursos a las abejas y pueden ser cultivadas con un fin económico determinado (Cucurbitáceas, algodón, alfalfa, tréboles, melilotus, cítricos, manzanos, perales, otros frutales, sauces, álamos, acacias, eucaliptos, etc.), o especies silvestres nativas o exóticas espontáneas. En general las abejas utilizan solamente una parte reducida de la flora presente, ya que no todas ofrecen un buen recurso, o son morfológicamente inadecuadas para ser explotadas por ellas, por ejemplo, es esencial la relación entre la profundidad de la corola y la longitud de la lengua, que permite extraer el néctar. (SAGP, 2012).

En el Perú la Ley N²6305 en su artículo 1 sostiene "Declárese de interés nacional a la Apicultura y la actividad agro-industrial de los productos apícolas por su importancia económica, social y ecológica, debiendo protegerse a la abeja doméstica-abeja Apis mellifera- y a las especies de abejas nativas como insectos útiles, así como a la flora apícola como riqueza nacional evitando su tala indiscriminada y propiciando su reforestación”. (El Peruano, 1994).

En la Región Amazonas actualmente no existe información sobre flora apícola, siendo necesario registrar las plantas existentes de uso apícola, para fomentar, su siembra y conservación, mejorando la producción de miel y productos derivados, pues existe actualmente una demanda insatisfecha de los mismos.

La importancia de la flora indicadora radica en que no todas las especies vegetales son de interés para la apicultura, en virtud que una especie puede ser muy nectífera pero esta especie puede tener baja ponderación en el número de individuos por hectárea. Por lo tanto, el aprovechamiento de una colmena tiene que estar relacionado con la cantidad y calidad de la flora. Existen especies vegetales que hacen un gran aporte, pero sus flores entregan poco néctar. (Raticelli, 2008).

Para determinar si una especie vegetal es importante desde el punto de vista de la apicultura es necesario considerar algunos aspectos como atractividad o intensidad de uso, fidelidad, abundancia, oportunidad de la floración, intensidad y longitud de la floración. (SAGP, 2012).

En el municipio de Icononzo, departamento del Tolima, Colombia, se realizó un estudio preliminar de la flora apícola que incluyó un inventario botánico, el análisis palinológico de las mieles y la elaboración de un calendario floral para la identificación de 74 plantas con atributos apícolas y la caracterización de sus periodos de floración y el aporte de recursos a la colmena. (Silva y Restrepo, 2012).

En el departamento de Antioquia, Colombia, la investigación definió un modelo para encontrar las especies de mayor importancia para la producción apícola. Esto permitió la identificación de las épocas de máxima densidad floral de 154 especies de interés para los productos de la colmena. Como principal aporte se determinaron los parámetros para establecer el nivel de importancia de la flora apícola, entre los que se encuentran: el reporte de especies apícolas en bibliografía especializada, distribución de las especies vegetales en la zona de estudio, abundancia relativa de cada especie, duración del periodo de floración, color de la flor, aromas florales, accesibilidad a la flor que tienen las abejas en un área de un metro cuadrado, tiempo de permanencia de la abeja en la flor, producto de la planta y concentración de azúcares en el néctar. Sin duda, este estudio se constituye en un referente importante en el trabajo con flora apícola y diferenciación de productos de la colmena. (Silva y Restrepo, 2012).

Posteriormente, en el departamento del Huila, Colombia se elaboraron calendarios florales de los municipios de La Argentina, Palestina y Pitalito. Estos contienen información que corresponde a las especies florecidas en un periodo de seis meses de seguimiento. De igual manera, este estudio incluyó observaciones sobre el comportamiento de forrajeo de las abejas y la importancia de algunas especies apícolas.

En el valle de San Andrés, Cuba, se evalúo relación de la población natural de abejas de la tierra (Melipona beecheii) y su flora, a través observaciones y muestreos realizados en las formaciones boscosas estudiadas. Se estudiaron las plantas melíferas y su utilización como sitios de nidificación de Melipona beecheii para cada una de las formaciones vegetales del valle San Andrés, también se analizaron los 
índices de diversidad y su relación con los valores de densidad de colonias. Se determinó que la densidad de colonias de abejas de la tierra está directamente relacionada con la riqueza y diversidad de especies de plantas melíferas encontradas en el área de estudio. Se comprobó además que las especies arbóreas más utilizadas como sitio de nidificación son: Bursera simaruba, Guazuma ulmifolia, Psidium guajava, Mangifera indica y Roystonea regia. (Ravelo, et. al., 2014).

En Argentina, el departamento Tala, provincia de Entre Rios, comprende el $12 \%$ de la producción apícola a nivel provincial. Actualmente se estima que en el departamento existen alrededor de 600 productores con rendimientos promedios de entre 30 a $40 \mathrm{~kg} / \mathrm{col} / \mathrm{año}$. La cosecha se extiende entre los meses de noviembre hasta abril con un pico en el mes de marzo, obteniendo mayoritariamente miel de chilca. Se observa un buen aporte floral primeramente de monte, dando una miel muy oscura que supera los $80 \mathrm{~mm}$ Pfund de color y posteriormente de la flor de chilca con rendimientos diferentes en distintas zonas del departamento, proporcionando una miel más clara. (Raticelli, 2008).

En el Perú existe gran diversidad de flora en toda la extensión del país; el cual permite obtener productos apícolas diferenciados, por ejemplo, mieles de algarrobo, nísperos, eucalipto, cítricos, etc. así como los multiflorales. Esto aunado a una política de incentivo y fomento a la producción orgánica significa una oportunidad de posicionar al Perú como proveedor confiable de productos diferenciados y de calidad. Mediante procesos y tecnología apropiada obtienen beneficios económicos al producir miel, polen, propóleo, cera y servicios de polinización, y es una actividad que ejerce sinergia con los cultivos, con la protección y conservación de la flora y los recursos forestales e incrementa la producción a través de la polinización. (MINAGRI, 2011).

Muchos son los apicultores que conocen a las abejas y su manejo correcto, el tratamiento adecuado de la miel, y muchos otros factores relacionados con la apicultura, pero ignoran por completo los procesos mediante los cuales las plantas producen néctar y polen. En general, los apicultores observaron que algunas plantas son más atrayentes que otras para las abejas, pero solo mediante una tarea sistemática de observación y clasificación puede señalarse cuáles especies son las más convenientes para cada localidad y época del año. (Root, 2008)

En los proyectos de forestación y reforestación, se puede incluir la siembra de plantas melíferas, basadas en la demanda mundial insatisfecha de miel y otros productos apícolas. (MINAGRI, 2011).

Las especies de interés apícola proveen de recursos a las abejas y pueden ser cultivadas con un fin económico determinado (cucurbitáceas, algodón, alfalfa, tréboles, melilotus, cítricos, manzanos, perales, otros frutales, sauces, álamos, acacias, eucaliptos, etc.), o especies silvestres nativas o exóticas espontáneas.

El Fundo Vitaliano ubicado a 2,450 m.s.n.m, se encuentra en la provincia de Chachapoyas región Amazonas, cuenta con gran variedad de recursos de fauna y flora. Inicia sus actividades apícolas en el año 2012, siendo la alimentación de las abejas el determinante de las características organolépticas de la miel, es que estas son alimentadas de los recursos florísticos de la zona. Las abejas tienen predilección por las flores de "salvia morada", "shisca", "tara" y "cadillo" entre las principales, motivo por el cual se hace necesario la identificación de plantas melíferas de la zona, para mejorar la alimentación de las abejas y aumentar la producción de miel y productos derivados.

Por las consideraciones antes vertidas y ante la demanda insatisfecha de productos apícolas, consideramos importante el estudio de la flora apícola en el Fundo Vitaliano, en la provincia de Chachapoyas región Amazonas, en el marco del estudio de la flora melífera de la región.

\section{MATERIAL Y MÉTODO}

Fundo Vitaliano, ubicado a 2,450 m.s.n.m., en el distrito de Chachapoyas, región Amazonas, tiene una superficie total de 102 hectáreas de tierra, que comprende diferentes formaciones vegetales como árboles, arbustos, hierbas.

La población estuvo constituida por la flora presente en el fundo Vitaliano, la muestra fue la flora utilizada por las abejas (flora apícola). Se realizaron muestreos mensuales de tipo intencional y selectivo. Se realizó la codificación de los especímenes muestreados.

Se ubicaron los apiarios existentes y después de localizadas las colmenas se marcaron transeptos radiales y se realizó el inventario de las especies de plantas de uso apícola a cada lado del transepto. (Pimentel y Ravelo, 2007).

La determinación de la época de floración de las plantas de uso apícola se realizó mediante revisión de fuentes bibliográficas, observaciones directas y encuestas (Pimentel y Ravelo, 2007), a los encargados del fundo Vitaliano.

Para la identificación de especies de flora apícola se procedió a la identificación taxonómica de las 
especies utilizando claves botánicas, información bibliográfica y la respectiva comparación con el material hebarizado en el Herbarium Truxillense de la Universidad Nacional de Trujillo (HUT).

Se determinó los azucares del néctar de las flores por la medición de los grados brix ( ${ }^{\circ}$ Brix), para ello se utilizó un refractómetro, (Salinas, et al.; 2013). Durante el periodo de floración, cuantificamos semanalmente el azúcar del néctar utilizando 15 flores por planta (Salinas et. al., 2013).

\section{RESULTADOS}

Tabla 1. Georeferenciación de los apiarios del Fundo Vitaliano.

\begin{tabular}{|c|c|c|c|}
\hline NoApiario & Coordenadas (UTM) & Altitud (msnm) & Observaciones \\
\hline \multirow{2}{*}{$1,2,3,4,5,5,6,7,8,9$} & 18M0181868 & \multirow[b]{2}{*}{2,406} & \multirow[b]{2}{*}{ Distancia 80m al norte } \\
\hline & 9313267 & & \\
\hline \multirow[b]{2}{*}{$10,11,12$} & 18M0181925 & \multirow[b]{2}{*}{2,424} & \multirow{2}{*}{$\begin{array}{c}\text { Distancia } 200 m \text { a l oeste (se tomó el } \\
\text { punto en el alfiafiar) }\end{array}$} \\
\hline & 9313633 & & \\
\hline \multirow[b]{2}{*}{13} & 18M0181899 & \multirow[b]{2}{*}{2,429} & \multirow{2}{*}{$\begin{array}{c}\text { Distancia } 100 \mathrm{~m} \text { a l nor este (se tomó } \\
\text { el punto en las fresas) }\end{array}$} \\
\hline & 9313698 & & \\
\hline \multirow[b]{2}{*}{$14,15,16$} & 18M0182162 & \multirow[b]{2}{*}{2,452} & \multirow{2}{*}{$\begin{array}{c}\text { Distancia } 200 \mathrm{~m} \text { al oeste (se tomó el } \\
\text { punto en los pinos) }\end{array}$} \\
\hline & 9313042 & & \\
\hline \multirow[b]{2}{*}{17,18} & 18M0182025 & \multirow[b]{2}{*}{2,460} & \multirow{2}{*}{$\begin{array}{l}\text { Distancia } 150 \mathrm{~m} \text { al oeste (se tomó el } \\
\text { punto en la casa de la entrada) }\end{array}$} \\
\hline & 9312790 & & \\
\hline
\end{tabular}

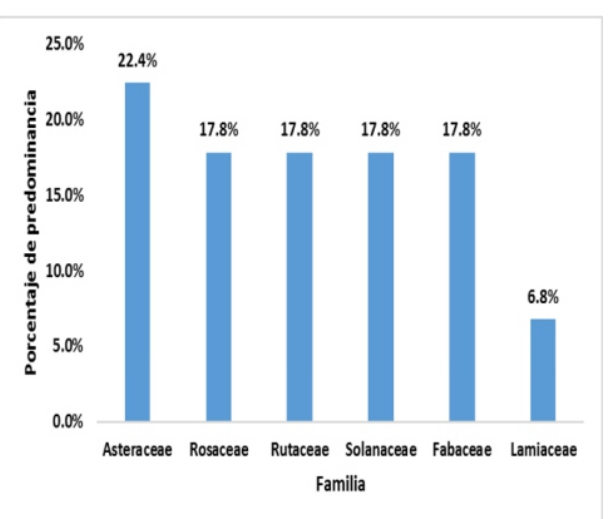

Gráfico 1. Porcentaje de flora apícola con mayor predominancia, según familias.

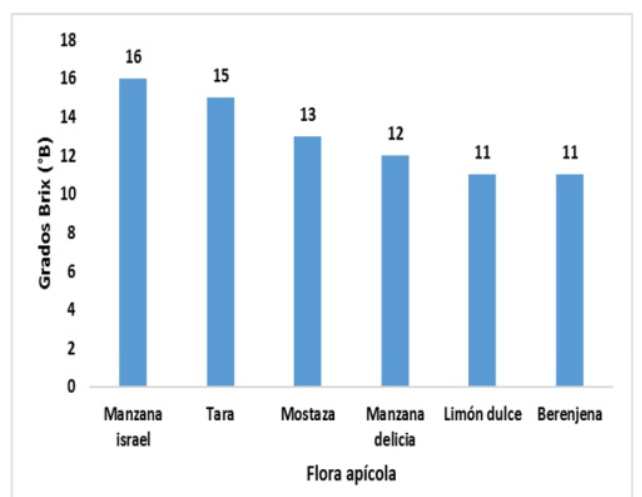

Grafico 2. Flora apícola con mayor valor de grados Brix.

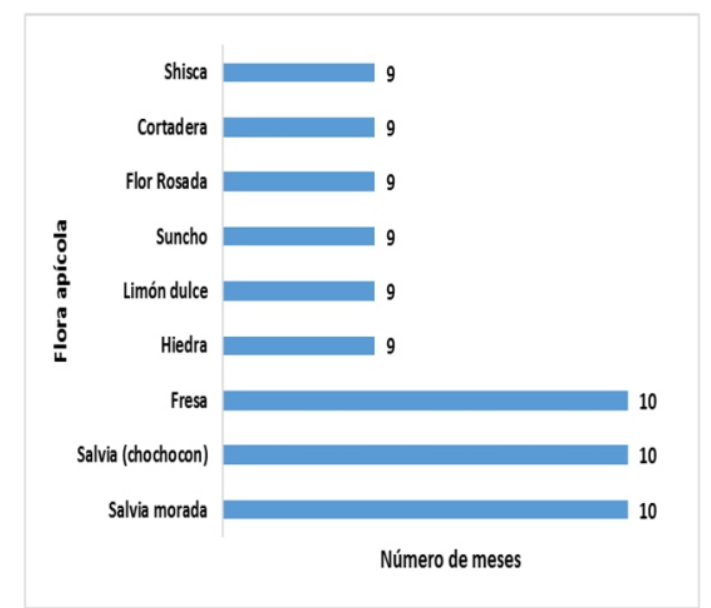

Grafico 3. Flora apícola con mayor número de meses de floración.

\section{DISCUSIÓN}

La flora de un lugar determina la producción apícola e influye sobre las características nutricionales y organolépticas de la miel de abejas (Insuasty, et. al, 2016), razón por el cual en nuestro estudio se georreferenciaron los apiarios del Fundo vitaliano, registrándose dieciocho apiarios (tabla 1).

En la Universidad de Nariño, Colombia cuando se identificaron y clasificaron taxonómicamente las especies florales se encontró que las familias botánicas con mayor interés apícola fueron Fabaceae y Asteraceae. (Insuasty, et. al, 2016). En otro estudio realizado en el municipio de Piendamó, Colombia sobre flora melífera se encontró que las familias botánicas de mayor importancia melífera fueron Asteraceae, Fabaceae, Mytaceae y Rutacae. (Montoya, et.al., 2017). Con el fin de construir una base para la formulación de futuras propuestas de investigación relacionadas con la apicultura en sistemas familiares y comerciales se buscó caracterizar algunas variables con el comportamiento de la abeja Apis mellifera, en aras de organizar el proceso productivo apícola para la obtención de miel de abejas en el apiario de la granja experimental Botana de la universidad de Nariño, se observó que Apis mellifera, visitó con mayor frecuencia las especies de Brassica rapa "nabo amarillo", Trifolium repens "trébol blanco", Taraxacum officinale "diente de león" y Trifolium pratense "trébol rojo" (Insuasty, et. al.; 2015). En nuestro estudio se encontró que el porcentaje de flora apícola con mayor predominancia, según familias fueron. Asteraceae, Rosaceae, Rutaceae, Solanaceae, Fabacae, Lamiaceae (gráfico 1).

En la Universidad de Nariño, se encontró taxonómicamente que las especies florales con potencial apícola; las especies vegetales de mayor 
Tabla 2. Clasificación taxonómica de flora apícola del fundo Vitaliano

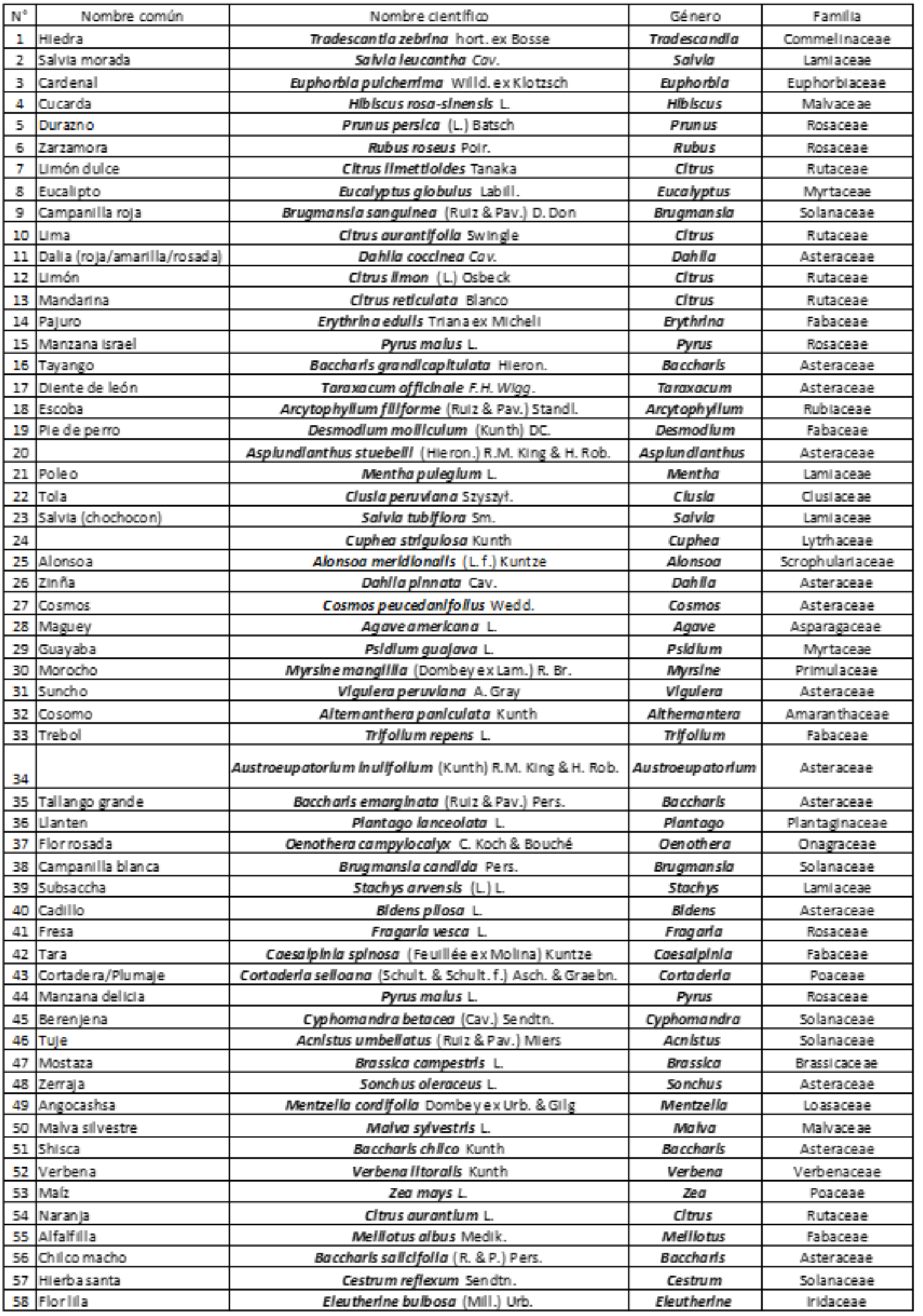


Tabla 3. Grados Brix de la flora apícola del Fundo Vitaliano.

\begin{tabular}{|c|c|c|c|}
\hline $\mathrm{N}^{0}$ & Nombre común & ${ }^{\circ}$ Brix & Nombre cientif ico \\
\hline 1 & Hiedra & $8^{\circ}$ & Tradescandia zebrina \\
\hline 2 & Salvia morada & $7.5^{\circ}$ & Salvia leucantha \\
\hline 3 & Cardenal & $3^{0}$ & Euphorbia pulcherrima \\
\hline 4 & Cucarda & $4^{\circ}$ & Hibiscus rosa-sinensis \\
\hline 5 & Durazno & $7^{\circ}$ & Prunus persia \\
\hline 6 & Zarzamora & $7^{0}$ & Rubus roseus \\
\hline 7 & Limón dulce & $11^{\circ}$ & Citrus limettioides \\
\hline 8 & Campanilla roja & $7^{\circ}$ & Brugmansia sanguinea \\
\hline 9 & Lima & $8^{\circ}$ & Citrus aurantifolia \\
\hline 10 & Dalia (roja/amarilla/rosada) & $10^{\circ}$ & Dahlia coccinea \\
\hline 11 & Limón & $5^{\circ}$ & Citrus limon \\
\hline 12 & Pajuro & $5^{\circ}$ & Erythrina edulis \\
\hline 13 & Manzana israe I & $16^{\circ}$ & Pyrus malus \\
\hline 14 & Tayango & $6^{\circ}$ & Baccharis grandecapitulata \\
\hline 15 & Diente de león & $8^{\circ}$ & Taraxacum officinale \\
\hline 16 & Escoba & $8^{\circ}$ & Arcythophyllum filiforme \\
\hline 17 & Pie de perro & $10^{\circ}$ & Desmodium molliculum \\
\hline 18 & Poleo & $7^{0}$ & Mentha pulegium \\
\hline 19 & Salvia (chochocon) & $4^{\circ}$ & Savia smithii/tubiflora \\
\hline 20 & & 6 & Cuphea strigulosa \\
\hline 21 & Zinña & $8^{\circ}$ & Dahlia pinnata \\
\hline 22 & Morocho & $6^{\circ}$ & Myrsine manglilla \\
\hline 23 & Suncho & $6^{\circ}$ & Viguiera peruviana \\
\hline 24 & Cosomo & $10^{\circ}$ & Althernantera paniculata \\
\hline 25 & Trebol & 9 & Trifolium repens \\
\hline 26 & & $6^{\circ}$ & Ophriosporus sp. \\
\hline 27 & Tallango grande & $10^{\circ}$ & Baccharis emarginata \\
\hline 28 & Llanten & $4^{\circ}$ & Plantago lanceolata \\
\hline 29 & Flor rosada & $3^{0}$ & Oenothera campylocalyx \\
\hline 30 & Campanilla blanca & $5^{\circ}$ & Brugmansia candida \\
\hline 31 & Subsaccha & $6^{\circ}$ & Stachys arvensis \\
\hline 32 & Cadillo & $8^{\circ}$ & Bidens pilos $a$ \\
\hline 33 & Fresa & $5^{\circ}$ & Fragaria vesca \\
\hline 34 & Tara & $15^{\circ}$ & Ceasalpina spinosa \\
\hline 35 & Cortadera/Plumaje & $1^{\circ}$ & Cortaderia selloana \\
\hline 36 & Manzana de licia & $12^{\circ}$ & Pyrus malus \\
\hline 37 & Berenjena & $11^{\circ}$ & Cyphomandra betacea \\
\hline 38 & Tuje & $7^{0}$ & Acnistus umbellathus \\
\hline 39 & Mostaza & $13^{\circ}$ & Brassica campestris \\
\hline 40 & Ze rraja & $8^{\circ}$ & Sonchus oleraceus \\
\hline 41 & Malva silvestre & $3^{0}$ & Malva hirsuta \\
\hline 42 & Shisca & $6^{\circ}$ & Baccharis chilca \\
\hline 43 & Verbena & $4^{\circ}$ & Verbena litoralis \\
\hline 44 & Alfalfilla & $8^{\circ}$ & Melilotus albus \\
\hline 45 & Chilco macho & $9^{\circ}$ & Baccharis salicifolia \\
\hline 46 & Flor li la & $5^{\circ}$ & Eleutherine bulbosa \\
\hline
\end{tabular}


Tabla 4. Calendario floral de la flora apícola del Fundo Vitaliano, en 10 meses de estudio de febrero a noviembre.

\begin{tabular}{|c|c|c|c|c|c|c|c|c|c|c|c|c|}
\hline \multirow[b]{2}{*}{$\mathrm{N}^{0}$} & \multirow[b]{2}{*}{ Nombre común } & \multirow[b]{2}{*}{ Nombre clent $|f| c o$} & \multicolumn{10}{|c|}{ Meses de floración } \\
\hline & & & Mes 1 & Mes 2 & Mes 3 & Mes 4 & Mes 5 & Mes 6 & Mes 7 & Mes 8 & Mes9 & Mes 10 \\
\hline 1 & Hiledra & Tro descontio zebrino & $\bar{x}$ & $\bar{x}$ & $\bar{x}$ & $\bar{x}$ & $\bar{x}$ & $\mathrm{x}$ & $\bar{x}$ & $x$ & $x$ & \\
\hline 2 & Salvia morada & Solvio leu contho & $\mathrm{x}$ & $\mathrm{x}$ & $\mathrm{x}$ & $\mathrm{x}$ & $x$ & $\mathrm{x}$ & $\mathrm{x}$ & $\mathrm{x}$ & $\mathrm{x}$ & $\mathrm{x}$ \\
\hline 3 & Cardenal & Euphorblo pulchemimo & $\mathrm{x}$ & $\mathrm{x}$ & $\mathrm{x}$ & $\mathrm{x}$ & $\mathrm{x}$ & $\mathrm{X}$ & & & $\mathrm{X}$ & \\
\hline 4 & Cucarda & Hibliscus roso-sinensis & $\bar{x}$ & $\bar{x}$ & $\bar{x}$ & $\mathrm{x}$ & $\bar{x}$ & $\bar{x}$ & & $\bar{x}$ & & $\bar{x}$ \\
\hline 5 & Durazno & Prunus persilo & & & & $\mathrm{x}$ & & $\mathrm{x}$ & $\mathrm{x}$ & $x$ & & $\mathrm{x}$ \\
\hline 6 & Zaramora & Rubus roseus & & & $\mathrm{x}$ & & & & $\mathrm{x}$ & $\bar{x}$ & $\bar{x}$ & $\mathrm{x}$ \\
\hline 7 & Limón dulce & Citrus limettioldes & $\mathrm{x}$ & $\mathrm{x}$ & $\mathrm{x}$ & & $\mathrm{x}$ & $\mathrm{x}$ & $\mathrm{x}$ & $\mathrm{x}$ & $\mathrm{x}$ & $\mathrm{x}$ \\
\hline 8 & Eucalipto & Eucolyptus globulus & $\mathrm{x}$ & & $\mathrm{x}$ & $\mathrm{x}$ & $\mathrm{x}$ & & $\mathrm{x}$ & & & \\
\hline 9 & Campanilla roja & Brugmansio songulneo & $\mathrm{x}$ & $\mathrm{x}$ & $\mathrm{x}$ & $\mathrm{x}$ & $\mathrm{x}$ & $\mathrm{x}$ & & $\mathrm{x}$ & $\mathrm{x}$ & $\mathrm{x}$ \\
\hline 10 & Lima & Cotus ourontifolio & & & $\mathrm{x}$ & & & & & & & \\
\hline 11 & Dalıa (roja/amarllla/rosada) & Dohllo cocclineo & $\mathrm{x}$ & $\mathrm{x}$ & $\mathrm{x}$ & & & & & & $\mathrm{x}$ & $x$ \\
\hline 12 & Limón & Otrus limon & & $\mathrm{x}$ & & $\mathrm{x}$ & $\mathrm{x}$ & & & $\mathrm{x}$ & & $\bar{x}$ \\
\hline 13 & Mandarina & Citrusreticuloto & & & & & & & & & & \\
\hline 14 & Pajuro & Erythrina edulis & $\mathrm{x}$ & $\mathrm{x}$ & & $\mathrm{x}$ & $\mathrm{x}$ & $\mathrm{x}$ & & & & $\mathrm{x}$ \\
\hline 15 & Manzana Israel & Pyrus malus & & $\mathrm{x}$ & $\mathrm{x}$ & $\mathrm{x}$ & $\mathrm{x}$ & $\mathrm{x}$ & & $\mathrm{x}$ & $\mathrm{x}$ & \\
\hline 16 & Tayango & Boccharis gran decopituloto & & & & & & & & & $\mathrm{x}$ & $\mathrm{x}$ \\
\hline 17 & Diente de león & Toroxocum officin ale & $\mathrm{x}$ & $\mathrm{x}$ & $\mathrm{x}$ & $\mathrm{x}$ & & & & $\mathrm{x}$ & $x$ & $\mathrm{x}$ \\
\hline 18 & Escoba & Arcyto phyllum filiforme & $\mathrm{x}$ & $x$ & $\mathrm{x}$ & $\mathrm{x}$ & $x$ & & $\mathrm{x}$ & $x$ & $\mathrm{x}$ & $\mathrm{x}$ \\
\hline 19 & Ple de perro & Desmodium molliculum & & & & & & & & & & $\mathrm{x}$ \\
\hline 20 & & Asplundionthus SP. & $\mathrm{x}$ & $\mathrm{x}$ & $\mathrm{x}$ & & & & & & & \\
\hline 21 & Poleo & Mentho puleglum & & & $\mathrm{x}$ & $\mathrm{x}$ & $\mathrm{x}$ & $\mathrm{x}$ & & & & \\
\hline 22 & Tola & Clusia peruviona & & & $\mathrm{x}$ & & & & & & & \\
\hline 23 & Salvia (chochocon) & solvio tubifiloro & $\mathrm{x}$ & $\mathrm{x}$ & $\mathrm{x}$ & $\mathrm{x}$ & $\mathrm{x}$ & $\mathrm{x}$ & $\mathrm{x}$ & $\mathrm{x}$ & $x$ & $\mathrm{x}$ \\
\hline 24 & & Cupheo striguloso & $\mathrm{x}$ & $\mathrm{x}$ & $\mathrm{x}$ & $\mathrm{x}$ & $\mathrm{x}$ & & & & & $\mathrm{x}$ \\
\hline 25 & Alonsoa & Alonsoo mendionolis & $\mathrm{x}$ & $\mathrm{x}$ & $\mathrm{x}$ & $\mathrm{x}$ & & & & & & $\mathrm{x}$ \\
\hline 26 & Znกิa & Dohlio pinnoto & $\mathrm{x}$ & $\mathrm{x}$ & $\mathrm{x}$ & $\mathrm{x}$ & & $\mathrm{x}$ & & $\mathrm{x}$ & $x$ & $\mathrm{x}$ \\
\hline 27 & Cosmos & Cosmos peuced onif ollus & $\mathrm{x}$ & $\mathrm{x}$ & $\mathrm{x}$ & $\mathrm{x}$ & & $\mathrm{x}$ & & & & $\mathrm{x}$ \\
\hline 28 & Maguey & Agove omericona & $\mathrm{x}$ & & $\mathrm{x}$ & & & & & & & \\
\hline 29 & Guayaba & Psidium guajovo & & $\mathrm{x}$ & $\mathrm{x}$ & & & & & & & \\
\hline 30 & Morocho & Myrsine mong lillo & & $\mathrm{x}$ & $\mathrm{x}$ & & & $\mathrm{x}$ & & & & $x$ \\
\hline 31 & Suncho & vigulero peruviono & $\mathrm{x}$ & $\mathrm{x}$ & $\mathrm{x}$ & $\mathrm{x}$ & $\mathrm{x}$ & $\mathrm{x}$ & $\mathrm{x}$ & $\mathrm{x}$ & $\mathrm{x}$ & \\
\hline 32 & Cosomo & A/thernontero poniculo to & $\mathrm{x}$ & & $\mathrm{x}$ & $\mathrm{x}$ & $\mathrm{x}$ & $\mathrm{x}$ & $\mathrm{x}$ & $\mathrm{x}$ & $\mathrm{x}$ & \\
\hline 33 & Trebol & Trifollum repens & $\mathrm{x}$ & $\mathrm{x}$ & $\bar{x}$ & $\mathrm{x}$ & $\mathrm{x}$ & & & $\mathrm{x}$ & & $\mathrm{x}$ \\
\hline 34 & & Ophriosporus sp. & & $\mathrm{x}$ & $\mathrm{x}$ & $\mathrm{x}$ & $\mathrm{x}$ & $\mathrm{x}$ & $\mathrm{x}$ & $x$ & $\mathrm{x}$ & \\
\hline 35 & Tallango grande & Boccharis emargin oto & & & $\mathrm{x}$ & $\mathrm{x}$ & & & & $\mathrm{x}$ & $\mathrm{x}$ & $\mathrm{x}$ \\
\hline 36 & Llanten & Plontago lancealo to & & & & & & & & $\bar{x}$ & & $\mathrm{x}$ \\
\hline 37 & Flor rosada & Deno thero compylocolyx & $\mathrm{x}$ & $\mathrm{x}$ & $\mathrm{x}$ & $\mathrm{x}$ & & $\mathrm{X}$ & $\mathrm{X}$ & $\mathrm{x}$ & $\mathrm{x}$ & $\mathrm{x}$ \\
\hline 38 & Campanilla blanca & Brugmansilo condida & $\mathrm{x}$ & $\mathrm{x}$ & $\mathrm{x}$ & $\mathrm{x}$ & & & $\mathrm{x}$ & $\mathrm{x}$ & $\mathrm{x}$ & \\
\hline 39 & Subsaccha & Stachys orvensis & $\mathrm{x}$ & $\mathrm{x}$ & $\mathrm{x}$ & $\mathrm{x}$ & & & & & & $\mathrm{x}$ \\
\hline 40 & Cadillo & Bidens plloso & $\mathrm{x}$ & $\mathrm{x}$ & $\mathrm{x}$ & $\mathrm{x}$ & & & & & & $\mathrm{x}$ \\
\hline 41 & Fresa & Frogorio vesco & $\mathrm{x}$ & $\mathrm{x}$ & $\mathrm{x}$ & $\mathrm{x}$ & $\mathrm{x}$ & $\mathrm{x}$ & $\mathrm{x}$ & $\mathrm{x}$ & $\mathrm{x}$ & $\mathrm{x}$ \\
\hline 42 & Tara & Ceosolpina spin oso & $\mathrm{x}$ & $\mathrm{x}$ & & & & $\mathrm{x}$ & $\mathrm{x}$ & & & $\bar{x}$ \\
\hline 43 & Cortadera/Plumaje & Corto derio selloono & & $\mathrm{x}$ & $\mathrm{x}$ & $\mathrm{x}$ & $\mathrm{x}$ & $\mathrm{x}$ & $\mathrm{x}$ & $\mathrm{x}$ & $\mathrm{x}$ & $\mathrm{x}$ \\
\hline 44 & Manzana delicla & Pyrus malus & $\mathrm{x}$ & $\mathrm{x}$ & & & & $\mathrm{x}$ & & $\mathrm{x}$ & $\mathrm{x}$ & \\
\hline 45 & Berenjena & Cyphomandro beto ceo & $\mathrm{x}$ & $\mathrm{x}$ & $\mathrm{x}$ & & & & & $\mathrm{x}$ & & $\mathrm{x}$ \\
\hline 46 & Tuje & Acnistus umbellothus & $\mathrm{x}$ & & & $\mathrm{x}$ & & & & $\mathrm{x}$ & $\mathrm{x}$ & \\
\hline 47 & Mostaza & Brossico compestris & $\mathrm{x}$ & $\mathrm{x}$ & $\mathrm{x}$ & & & & & $\mathrm{x}$ & & \\
\hline 48 & zerraja & Sonch us aleraceus & $\mathrm{x}$ & $\mathrm{x}$ & $\mathrm{x}$ & & & & & $\bar{x}$ & & $\mathrm{x}$ \\
\hline 49 & Angocashsa & Mentzelio cordifolia & $\mathrm{x}$ & $\mathrm{x}$ & $\mathrm{x}$ & $\mathrm{x}$ & & & & & & \\
\hline 50 & Malva sillvestre & Molvo hirsuto & NR & $\mathrm{x}$ & $\mathrm{x}$ & $\mathrm{x}$ & $\mathrm{x}$ & & $\mathrm{x}$ & $\mathrm{x}$ & & \\
\hline 51 & Sh isca & Bocchoris chilco & NR & $\mathrm{x}$ & $\mathrm{x}$ & $\mathrm{x}$ & $\mathrm{x}$ & $\mathrm{x}$ & $\mathrm{x}$ & $\mathrm{x}$ & $\mathrm{x}$ & $\mathrm{x}$ \\
\hline 52 & Verbena & Verben a lito ralis & NR & $\mathrm{x}$ & $\mathrm{x}$ & & & & & $\mathrm{x}$ & $\mathrm{x}$ & $\mathrm{x}$ \\
\hline 53 & $\mathrm{Ma} / 2$ & Zeo mays & NR & NR & $\mathrm{x}$ & $\mathrm{x}$ & & & & & & \\
\hline 54 & Naranja & Cl trus aurantium & NR & NR & NR & NR & NR & NR & & & & \\
\hline 55 & Alfalfilla & Mellibtus albus & NR & NR & NR & NR & NR & NR & $\mathrm{X}$ & $\mathrm{X}$ & $\mathrm{X}$ & $\mathrm{x}$ \\
\hline 56 & Chilco macho & Bocchoris solicifollo & NR & NR & NR & NR & NR & NR & $\mathrm{x}$ & $\mathrm{x}$ & $\mathrm{x}$ & \\
\hline 57 & Hile rba santa & Cestrum sp. & NR & NR & NR & NR & NR & NR & $\mathrm{x}$ & $x$ & & \\
\hline 58 & Flor lila & Eleutherine bulbosa & NR & NR & NR & NR & NR & NR & $\mathrm{x}$ & $\mathrm{x}$ & $\mathrm{x}$ & \\
\hline
\end{tabular}

Leyenda:

NR: No registrado

$\mathrm{X}$ : Presenta floración

$\square$ : No presenta floración 
importancia identificadas según la clasificación binomial fueron Brassica rapa, Traxacum officinale, Trifolium repens, Fragaria chiloensis, Eucalyptus globulus (Insuasty, et. al, 2016). El estudio en el municipio de Piendamó, Colombia, se colectaron 150 plantas pertenecientes a 30 especies, 24 géneros y 13 familias, siendo las más representativas las Asteráceas con un 20\% y Fabaceas en un 20\% (Montoya, et.al., 2017). En nuestra investigación se colectaron 58 plantas pertenecientes a 58 especies, 48 géneros y 24 familias. La familia que tuvo mayor predominancia fue la Asteraceae con un 22.4\%. (tabla 2).

Cuando se estudió la flora melífera municipio de Piendamó, Colombia se encontró que el néctar de las flores tenían valores entre los 30 y $43{ }^{\circ}$ Brix. (Montoya, et.al., 2017). En nuestro estudio las plantas que tubieron mayor grado Brix $\left({ }^{\circ} \mathrm{B}\right)$ fueron Pyrus malus "manzana israel" y Ceasalpina spinosa "tara", con valores de $16^{\circ} \mathrm{B}$ y $15^{\circ} \mathrm{B}$, respectivamente. (tabla 3 y gráfico 2).

La elaboración de un calendario floral es importante porque es una herramienta para la planificación de la actividad apícola porque contiene información sobre las épocas de floración (Silva y Restrepo, 2012). En nuestra investigación la flora apícola con diez meses de floración, de febrero a noviembre correspondió a Salvia leucantha "salvia morada", Salvia tubiflora "salvia-chochocon" y fragraria vesca "fresa" (gráfico 3 y tabla 4).

\section{CONCLUSIONES}

Existen 24 familias y 48 géneros de flora apícola en el Fundo Vitaliano.

La familia que tiene mayor predominancia es la Asteraceae con un $22.4 \%$.

Las plantas que tienen mayor grado Brix $\left({ }^{\circ} \mathrm{B}\right)$ son Pyrus malus "manzana israel" y Ceasalpina spinosa "tara", con valores de $16^{\circ} \mathrm{B}$ y $15^{\circ} \mathrm{B}$, respectivamente.

La flora apícola con diez meses de floración corresponde a Salvia leucantha "salvia morada", Salvia tubiflora "salvia-chochocon" y fragraria vesca"fresa".

\section{REFERENCIAS BIBLIOGRÁFICAS}

El Peruano. (1994). Ley $N^{\circ} 26305$. Publicado en el diario oficial EL Peruano el 13 de mayo de 1994.

Insuasty, E. Martinez,J. y Jurado, H. (2015). Evaluación del proceso productivo apícola, basado en la caracterización etológica de la abeja Apis mellifera. Rev. Veterinaria y Zootecnia. 9(1), 01-15.
Insuasty, E. Martinez,J. y Jurado, H. (2016). Identificación de flora y análisis de miel de abeja para la producción apícola. Rev. Biotecnología en el sector Agropecuario y Agroindustrial. 14 (1), $37-44$.

Montoya, B.; Baca, A. y Bonilla, B. (2017). Flora melifera y su oferta de recursos en cinco veredas del Municipio de Piendamó, Cauca. Rev. Biotecnología en el Sector Agropecuario y Agroindustrial. Edición Especial $N^{\circ} 1,20-28$.

Ministerio de Agricultura. MINAGRI. (2011). Propuesta del Plan Nacional de Desarrollo Apícola. Lima, Perú: Comisión multisectorial de naturaleza temporal adscrita al Ministerio de Agricultura encargada de elaborar la propuesta de Plan Nacional de Desarrollo Apícola. Resolución Suprema $\mathrm{N}^{\circ} 156-$ 2011-PCM.

Pimentel, O. y Ravelo, K. (2007). Flora melifera y densidad de abejas de la tierra. Rev. Apitec. 1(2), 34-36.

Raticelli, F. (2008). Flora apícola indicadora del Departamento de Tala. Recuperado de http:/www.monografias.com/trabajos59/ flora-apicola/flora-apicola2-shtml.

Ravelo, K., Hernández, F., Panaque, I., Toledo, L. y Gutiérrez, H. (2014). Relación de la población natural de abejas de la tierra (Melipona beecheii) con la flora en el valle San Andrés. Revista Cubana de Ciencias Forestales, 2(1), 50-66.

Root, A. (2008). ABC y XYZ de la apicultura. Buenos Aires, Argentina: Editorial Hemisferio Sur S.A.

Secretaria de Agricultura, Ganadería, Pesca y Alimentos de la Nación Argentina. SAGP. (2012). Flora apícola. $\mathrm{R} \mathrm{e} \mathrm{c} \mathrm{u} \mathrm{p} \mathrm{e} \mathrm{r} \mathrm{a} \mathrm{d} \mathrm{o} \mathrm{d} \mathrm{e}$ http:/www.agrobit.com/info_tecnica/alte rnativos/apicultura/AL_000003ap.htm

Silva, L. M. y Restrepo, S. (2012). Flora apícola: Determinación de la oferta floral apícola como mecanismo para optimizar producción, diferenciar productos de la colmena y mejorar la competitividad. Bogotá, Colombia: Humboldt.

Salinas, L.; Arismendi, C.; Domínguez, C.; Castillo, C ; Lara, C. (2013). Producción de néctar 
y flores como rasgos compensatorios al robo de néctar en Bouvardi ternifolia

(Rubiaceae): Experimentos de campo

(México). Revista Botanical Sciencies

91 (1), 85-92. 